\title{
Energy and Hop count aware Ant based Routing Protocol for Wireless Sensor Networks
}

\author{
Chaganti B N Lakshmi ${ }^{1}$, Dr. S. K. Mohan Rao ${ }^{2}$ \\ Associate Professor, Mahaveer Institute of Science and Technology, Hyderabad, Telangana, India ${ }^{1}$ \\ Professor, Gandhi Institute for Technology, Bhuvaneswar, Odissha, India ${ }^{2}$
}

\begin{abstract}
Maximizing the lifetime of a Wireless Sensor Networks is an NP hard combinatorial optimization problem. Such problems can not be solved within polynomially bounded time. A near optimal solution can be obtained in a relatively short time by applying heuristics. Ant Colony Optimization which is a meta heuristic inspired from the behaviour of real ant colonies is applied here to find an optimal routing path and hence to increase the network lifetime. The proposed protocol finds the path to transmit the data based on the energy remained in the sensor nodes and the number of hops required to reach the node. The performance of the protocol is compared with the existing Ad hoc on demand Distance Vector routing protocol and Self Healing Routing Protocols for Wireless Sensor Network in terms of energy consumption, average delay, packet delivery ratio and packet drop.
\end{abstract}

Keywords: Ant Colony Optimization, Residual Energy, Transmission Range, Wireless Sensor Network.

\section{INTRODUCTION}

A Wireless Sensor Network (WSN) is a special kind of Mobile ad hoc network consisting of ample number of small sized sensor nodes where each sensor node contains one or more sensors to sense the given physical phenomenon[1 ]. Not only sensing unit, the Sensor Node also consists of a processing unit for computations, a memory unit to store the data and a transmission unit to send or receive the sensed data. All the units of a Sensor Node operate on limited battery power supply. Since the Wireless Sensor Networks are mainly deployed in unattended environments like terrestrial areas, forests etc, these batteries cannot be recharged or replenished frequently[2]. So, the power energies should be utilized properly in order to increase the network lifetime.

\section{A. Network Lifetime}

Generally the lifetime of a network is treated as the time period from the operational state of the network to in operational state of the network. But in case of WSNs, it is application specific[3]. For some applications the lifetime is considered as the time till the first sensor node dies and for some other applications it is the time till some percentage of sensor nodes die or for some other applications it is considered as the time till the network partitions into disjoint subnets and for some other applications it is considered as the time till all the sensor nodes in the network die and for some other applications it is considered as the time till the number of packets delivered falls below a threshold value. In this way, the definition of lifetime varies from application to application. For any application, the main objective of the WSN is increasing the network lifetime, since WSNs are generally deployed in harsh and hostile environments and the limited batteries cannot be replenished or recharged frequently[4]. Maximizing the network lifetime is proved as NP hard combinatorial optimization problem. So, it can not be solved easily to optimality within a polynomially bounded time but near optimal solutions can be obtained with the application of heuristics. Since energy consumption and network lifetime are inversely related to each other, minimizing the energy consumption may lead to maximum network lifetime.

\section{B. Fault Tolerance}

Faults encounter more frequently in WSNs due to many reasons such as power depletion of Sensor Nodes, physical damage, unreliable wireless links, environmental effects and so on. These faults are to be identified and appropriate measures are to be taken to get the desired level of functionality[5]. Fault detection mostly depends on type of application and type of fault. Faults can be identified either in centralized approach or distributed approach. Since the Sensor Nodes are more resource constrained, centralized approaches are not suitable for WSNs. With distributed approach the fault detection capability is evenly distributed to all the nodes of WSN. The WSN lifetime can be improved by applying suitable fault tolerant mechanism depending on the application.

\section{Ant Colony Optimization}

Ants lay down a substance called pheromone while they are searching for the food from their nest. During this process, shorter paths will get more pheromone compared to longer paths. Slowly, all the ants converge to the shorter paths having more pheromone. Ant Colony Optimization works on this same principle which is inspired from the foraging behaviour of real ants and is introduced by Morco Dorigo in 1999. Ant Colony Optimization (ACO) is a meta heuristic in which a colony of artificial ants cooperate in finding optimal solutions to different static 
Vol. 6, Issue 1, January 2017

and dynamic difficult combinatorial optimization problems[6]. The applications of ACO include Routing problems (Ex: traveling salesperson problem, Vehicle
Routing Problem), multiple knapsack problem, graph colouring problem, Quadratic assignment problem, Network Routing problems (Connection oriented and Connection less Routing) etc.

The remainder of the paper is organized as follows: Section II reviews few of the existing ant based routing methods to improve WSN lifetime. Section III describes the proposed routing protocol :Ant colony Optimization based Routing Protocol (ACORP). In section IV, the performance of the protocol is evaluated and compared with Ad hoc On demand Distance Vector routing protocol AODV and Self Healing Routing Protocol (SHRP) and the results are showed. Finally, Conclusion is presented at the end.

\section{RELATED WORK}

WSN lifetime can be increased in various methods such as: usage of different data aggregation techniques, implementing various clustering strategies, employing different power saving mechanisms like Dynamic Power Management (DPM), deploying a few resource rich mobile nodes in addition to simple nodes, by using mobile sink, large deployment of SNs in the target area, knowing the location information of neighbour nodes and sink, employing energy efficient routing protocols etc.

In [7] $\mathrm{ACO}$ is used in the computation of energy field within the sink range and in calculating the next best location for the sink by sending ants. When the new location of sink is better than the current location, sink is moved to that position. Here, the network lifetime is limited by the holes created by the energy depleted nodes around the sink and it can enhance lifetime only when the load is distributed equally.

In [ 8] proposed three improvements to the original Energy Efficient Ant Based Routing algorithm. They are :1. In the initialization of the routing tables, priority is given to neighbouring nodes that simultaneously could be the destination 2. Up dation of routing tables in case of a node or link failure 3 . Reduced the flooding ability of ants for congestion control. Even though it is more energy efficient the time for convergence is more and complexity is more in updating the routing tables. In [9] Search angle is used to limit the search area of ants to Max Min Ant System algorithm. After each round, pheromone updation takes place by best ants which give sub optimal solution. With this the convergence speed is increasing but it is not supporting mobility of Sensor nodes.

In [10 ] multiple paths are found between source and sink using ACO mechanism. The best path is evaluated based upon the current residual energy of sensor $\mathrm{y}$, minimum residual energy of sensors visited by Ant-F, the average residual energy of the route from the sender $\mathrm{x}$ to $\mathrm{y}$ and the path length up to $y$. Here Energy balancing is achieved but Routing overhead is more due to ants flooding.

\section{III.PROPOSED ROUTING PROTOCOL}

Two kinds of ants are used in ACO mechanism and they are Forward Ant (FANT) and Backward ANT (BANT). At regular intervals, from every network node s, a forward ant FANT is launched towards a randomly selected destination node. While travelling towards their destination nodes, the FANTs store the address of each visited node Nk and the Residual Energy remained in that node, Hop length to reach to that node in a memory stack. At each node $\mathrm{k}$, each FANT probabilistically chooses the next node based on a greedy stochastic policy. If a cycle is detected, that is, if the ant is forced to return to an already visited node, the cycle's nodes are popped from the ant's stack and all memory about the cycle is destroyed. When the destination node $\mathrm{d}$ is reached, the FANT generates a BANT. The FANT transfers all the memory contained in the stack to the BANT, and dies. The BANT takesthe same path as the corresponding FANT, but in the opposite direction. At each node k, the BANT pops the stack to move to the next node. The BANT updates the pheromone while coming to source and updates routing table once it reaches to source. The algorithm for the proposed ACORP is represented below.

\section{Algorithm ACORP}

1. Start

2. Initialize $t=0$,No.of.Cycles $=0, \tau_{i j}(t)=$ constant

3.Place $m$ Fants on $n$ nodes

4. Next hop Selection:

$$
P_{i, j}^{k}(t)= \begin{cases}\frac{\left[\tau_{i j}(t)\right]^{\alpha}\left[\eta_{i j}\right]^{\beta}}{\sum_{k \in \text { allowed } k}\left[\tau_{i k}(t)\right]^{\alpha}\left[\eta_{i k}\right]^{\beta}} & \text { if } j \in \text { allowed } k \\ 0 & \text { otherwise }\end{cases}
$$

5. Pheromone Updation

$$
\tau_{\mathrm{ij}}(\mathrm{t}+1)=\frac{\tau_{\mathrm{ij}}(\mathrm{t})+\Delta \tau_{\mathrm{ij}}}{1+\Delta \tau_{\mathrm{ij}}} \quad \text { for the selected node } \mathrm{j}
$$

6.Repeat the steps 4 and 5 until Fant reaches destination

7. At destination, create Bant using the information supplied by Fant.

8. movement of Bant until it reaches source

9. Set $\mathrm{t}=\mathrm{t}+1$, No.of.Cycles ++ ;

10. Repeat the steps from 3 to 9 until network stagnates or No.of Cycles=Maximum No.of Cycles 11.Stop

The source selects the path containing minimum hop count and maximum residual energy from the route table. Source uses the selected path as the primary path for data transmission. 


\section{IV.SIMULATION STUDY}

NS2 is an object-oriented, discrete event-driven network simulator developed at UC Berkeley written in $\mathrm{C}++$ and Otcl. NS2 is a publicly available common simulator which supports for simulating of a large number of protocols[11]. It provides a very rich infrastructure for developing new protocols. It also provides the opportunity to study largescale protocol interaction in a controlled environment. To study and evaluate the proposed protocol ACORP, NRL sensor sim is used, since it is developed using NS-2 which is an open source software.

The performance of proposed ACORP mechanism is compared with the Ad hoc On Demand Distance Vector Routing method and SHRP. Mainly the performance is evaluated according to the metrics: Packet delivery ratio, Average end-to-end-delay, Average energy consumption and Packet Drop. In the experiment the transmission range of the nodes is varied as $250,300,350$ and $400 \mathrm{~m}$ The simulation time considered is $50 \mathrm{sec}$. The size of the area is $500 * 500$. Constant Bit Rate traffic source and MAC 802.11 is considered.

The end-to-end-delay is averaged over all surviving data packets from the sources to the destinations. Packet delivery ratio is the total number of packets received by the receiver during the transmission. Packet drop is the number of packets dropped during the data transmission. Energy consumption is the average energy consumed by the nodes in receiving and sending the packets.

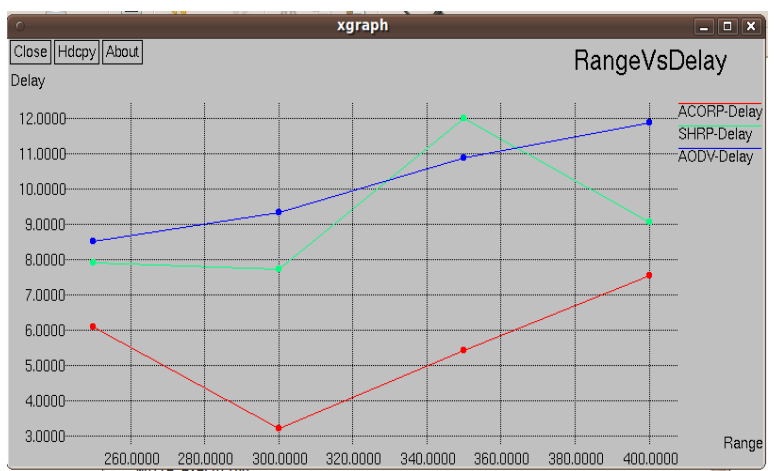

Fig. 1 Range Vs Delay

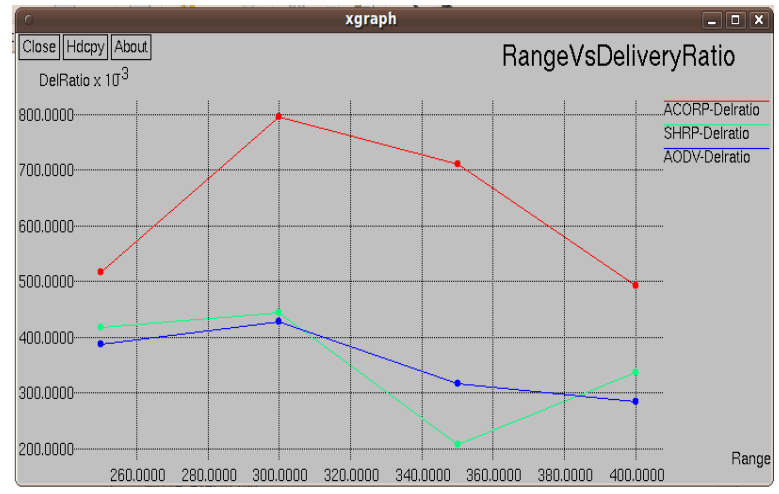

Fig. 2 Range Vs Delivery Ratio

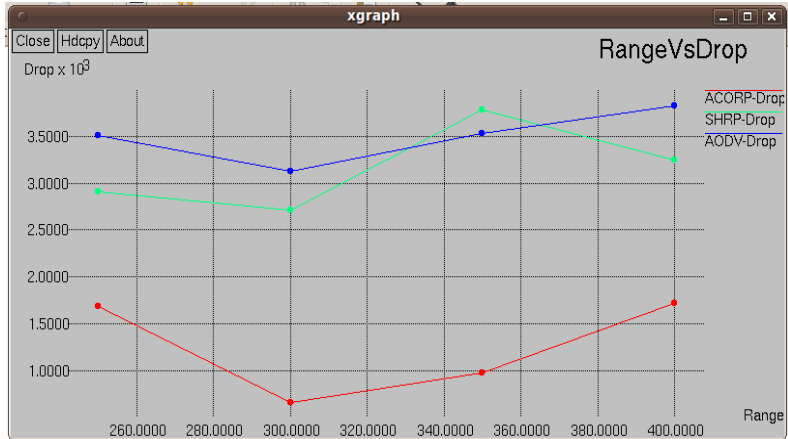

Fig. 3 Range Vs Drop

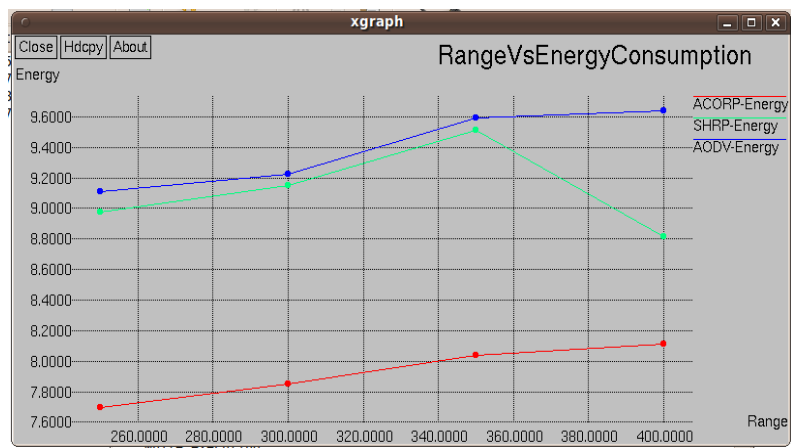

Fig. 4 Range Vs Energy Consumption

\section{CONCLUSION}

The performance of the proposed protocol ACORP is observed in terms of packet Delay, packet Delivery ratio, energy consumption and packet drop by varying the transmission power of the nodes. It is observed that the proposed ACORP is giving good results than the AODV and SHRP.

\section{REFERENCES}

[1] I.F. Akyildiz, W. Su, Y. Sankarasubramaniam,and E. Cayirci "Wireless sensor networks: a survey", elseiver publications : Computer networks, vol.38,no.4,pp.393-422, 2002

[2] Kemal Akkaya and Mohamed Younis, "A Survey on Routing Protocols for Wireless Sensor Networks", Adhoc Networks, Volume 3, issue 3, pp.325-349, May 2005

[3] Dong Q. Maximizing System Lifetime in Wireless Sensor Networks. Proceedings of the Fourth International Symposium on Information Processing in Sensor Networks; Los Angeles, CA, USA. 25-27 April 2005; pp. 13-19.

[4] Shio Kumar Singh, M P Singh and D K Singh, "Routing Protocols in Wireless Sensor Networks -A Survey" International Journal of Computer Science \& Engineering Survey (IJCSES) Vol.1, No.2, pp.63-83, November 2010.

[5] Luciana Moreira Sa de Souza, Harald Vogt and Michael Beigl," A survey on fault Tolerance in Wireless Sensor Networks", IJLTC, Latest trends in computing, Vol 1, Issue2, Dec-2010

[6] Dorigo, M.; Birattari, M.; Stutzle, T.; , "Ant colony optimization," Computational Intelligence Magazine, IEEE , vol.1, no.4, pp.28-39, Nov. 2006

[7] Amandeep Singh, Sunny Behal,"Ant Colony Optimization for improving network lifetime in Wireless Sensor Networks" In International Journal of Engineering Sciences, Vol.8, June2013

[8] A. M. ZungeruKah Phooi, SengLi-Minn AngW.C. Chia, "Energy efficiency performance improvements for ant based routing algorithm in Wireless Sensor Networks" Journal of Sensors, Article 759654, 2013 
[9] Li Luo and Layuan Li, "An Ant Colony System based Routing Algorithm for Wireless Sensor Network" in proceedings of IEEE International conference on Computer Science and Electronics Engineering, DOI 10.1109/ICCSEE.2012.145, 2012

[10] Ahmed M. Shamsan Almshreqi, Borhanuddin Mohd Ali,, Mohd Fadlee A. Rasid, Alyani Ismail and Pooria Varahram, "An Improved Routing mechanism using Bio-Inspired for Energy Balancing in Wireless Sensor Networks", In proceedings of IEEE ICOIN, DOI.978-1-4673-0250-0,2012.

[11] Mrs. Poonam Chhimwal1, Dhajvir Singh Rai2, Deepesh Rawat3," Comparison between Different Wireless Sensor Simulation Tools", IOSR Journal of Electronics and Communication Engineering (IOSR-JECE) e-ISSN: 2278-2834,p- ISSN: 2278-8735. Volume 5, Issue 2 (Mar. - Apr.2013), PP54-60

\section{BIOGRAPHIES}

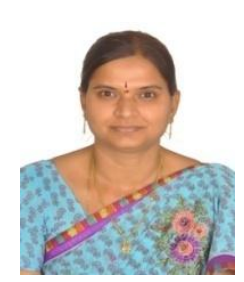

Chaganti B N Lakshmi received her B.Tech and M.Tech in Computer Science and Engineering from Jawaharlal Nehru Technology University,Hyderabad. She is pursuing her Ph.D from Rayalaseema University, Kurnool, Andhra Pradhesh. She is a Member of IETE, ISTE, Sensor Research Society (SRS) and Member of Computer Society of India (CSI). Her research interests include Advanced Computer Networks, MANETs and Sensor Networks.

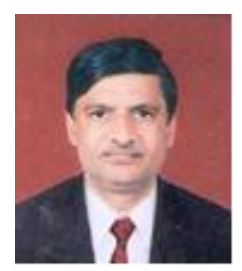

Dr. S Krishna Mohan Rao received B.Tech degree from JNTU, Hyderabad, M.Tech in Power Systems from JNTU, Ananthapur, M.Tech in Computer Science from IETE, and Ph.d in Computer Science and Engineering from Osmania University in 2009. He has 19 years of Industrial Experience, both in India and abroad and 8 years of teaching and research experience. He has published 6 papers in International Journals and 8 in National Journals. His research areas are Wireless Networks, Mobile Adhoc Networks, Industrial Management and Data ware House and Data Mining. He is Fellow of IETE, IE(I) and Life member of ISTE. 